\title{
Parte II. Enfermedad de Chagas en el adulto, la infancia y adolescencia
}

\author{
Comité de Parasitología, Departamento de Enfermedades Emergentes y Re-emergentes, \\ Ministerio de Salud de Chile*
}

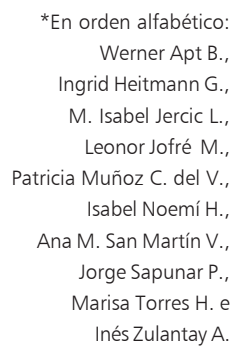

\section{Part II. Chagas disease in adults, infancy and adolescence}

The different stages of Chagas disease in adults: acute, undetermined or latent and chronic phases are described. This document contains guidelines for etiological diagnosis of Chagas disease and its treatment. In chronic phase, as cardiac and digestive system (esophagus and colon) are affected, symptoms and signs, evolution of the disease, laboratory analysis and treatment are described. The following topics in congenital Chagas disease are boarded: its prevalence in pregnant women, the importance of mother phase of disease, repercussions of the parasite transmission to the fetus, the frequency of transmission, how the infection to the fetus is produced, the importance of chronic infection in consecutive pregnancies, and clinical consequences to the newborn infant including symptoms of congenital disease. Concomitance with human immunodeficiency virus is commented. No vertically transmitted Chagas disease in infancy and adolescents has similar clinical manifestations as in adults. Direct and indirect laboratory tests of infection are described and an algorithm for diagnosis and follow up of vertical transmission of Trypanosoma cruzi is presented.

Key words: Chagas disease, clinical features, congenital Chagas.

Palabras clave: Enfermedad de Chagas, manifestaciones clínicas, Chagas congénito.

\section{Enfermedad de Chagas en el adulto}

$\mathrm{L}$ a enfermedad de Chagas presenta características regionales. En algunas zonas de Argentina, son frecuentes los cuadros agudos con signo de Romaña-Mazza, en tanto que en Chile, esto es excepcional. En el período crónico, dentro de los megasíndromes digestivos, el megacolon es más frecuente en nuestro país. En Brasil, se encuentra con mayor frecuencia el compromiso esofágico (megaesófago). En Venezuela, en un comienzo se pensó que sólo existía la cardiopatía y no los megasíndromes digestivos; sin embargo, hoy se reconocen ambas entidades.

La enfermedad presenta tres períodos bien definidos: fase aguda, fase crónica indeterminada o latente y fase crónica determinada. El período de incubación en la fase aguda es de 4 a 10 días y de menor duración cuando la vía de adquisición es transfusional.

\section{Período agudo}

Generalmente es asintomático, y más frecuente en personas jóvenes. Se evidencia una alta parasitemia, con síntomas y signos transitorios. Este período se extiende por dos a cuatro meses. Alrededor del 1 a $2 \%$ de los casos de enfermedad de Chagas se pesquisan en esta etapa. Los pacientes agudos sintomáticos pre- sentan: fiebre, signos de puerta de entrada (ver siguiente párrafo), edema, adenopatías satélites, hepatomegalia y esplenomegalia. La fiebre es frecuente, irregular, pero puede ser continua y alta. Se acompaña de anorexia, astenia, mialgias, cefalea y ocasionalmente artralgias. El cuadro febril suele persistir por un período de dos a cuatro semanas.

Los signos de puerta de entrada o chagomas de inoculación, son lesiones cutáneas, más frecuentes en la cara y extremidades por ser los sitios más expuestos a las vinchucas. Muy típico es el de la región ocular llamado signo de Romaña-Mazza, que se presenta como edema bipalpebral, unilateral, de color rosado violáceo claro, indoloro y duro. Existe aumento de tamaño de la glándula lagrimal accesoria (dacrioadenitis) y adenopatía satélite, los ganglios más comprometidos son los preauriculares, no adheridos a los tejidos adyacentes, algo sensibles y duros. Los chagomas pueden presentarse en cualquier parte de la piel, con aspecto furúnculoideo y de color rosado violáceo e indurados; tienen una duración variable, la que puede extenderse hasta 15 días.

En este período, el compromiso cardíaco se presenta como una miocarditis: el paciente presenta taquicardia e hipotensión, a veces existe ritmo de galope. Algunos casos pueden llegar a la insuficiencia cardíaca congestiva. El electrocardiograma puede evidenciar 
taquicardia sinusal, y alteraciones de la onda T. Se presenta hepatomegalia en $40 \%$ de los casos y compromiso meningo-encefálico, más frecuente en niños menores, representando un índice de gravedad. Entre los exámenes de laboratorio destaca una VHS elevada y puede encontrarse la PCR elevada.

Pronóstico

Es de curso habitualmente benigno en inmunocompetentes. La tasa de letalidad es de 2 a $7 \%$.

\section{Antecedentes epidemiológicos}

Son de importancia para plantear el diagnóstico.

Exámenes de laboratorio (ver parte V: Diagnóstico de laboratorio)

- Búsqueda del Trypanosoma cruzi en sangre por examen al fresco entre lámina y laminilla, gota gruesa y el método de Strout (material centrifugado).

- Búsqueda de anticuerpos de tipo IgG e IgM.

- Xeno-diagnóstico.

- RPC y RPC a tiempo real (cuantitativa) para enfermedad de Chagas.

- Excepcionalmente, pesquisa de T. cruzi en biopsias de tejidos e inoculación experimental.

Tratamiento de la etapa aguda de enfermedad

El tratamiento etiológico debe iniciarse precozmente, ya que con esto se evita el paso a la fase crónica. Los fármacos antiparasitarios disponibles presentan mayor efectividad en este período. Duración mínima: 30 a 60 días, según el fármaco (ver Parte VI: Tratamiento de la enfermedad de Chagas). Es conveniente indicar reposo con el propósito de evitar la miocarditis.

\section{Período crónico indeterminado o latente}

Representa entre 50 y $70 \%$ de todos los pacientes chagásicos. Se caracteriza por la ausencia de síntomas cardíacos, digestivos, etc. Los pacientes tienen parasitemia y serología positiva (títulos de IgG bajos), pero otros exámenes de laboratorio son normales, tales como: electrocardiograma y radiografías. Esta forma persiste, por lo menos en $30 \%$ de los chagásicos, durante toda su vida. El resto puede evolucionar a una forma crónica determinada, en un lapso de 10 a 30 años. En Chile, 2\% de los pacientes podrían anualmente pasar de la forma crónica indeterminada a cardiopatía crónica. En Brasil, este porcentaje es menor.

\section{Período crónico determinado}

Las formas crónicas determinadas son principalmente la cardiopatía, colopatía y esofagopatía. El compromiso de otros órganos es infrecuente, tales como estómago, duodeno, vejiga, uréteres, etc. Estas formas de presentación pueden ocurrir separadamente o co- existir en un mismo enfermo. En esta etapa existe una parasitemia baja, con títulos elevados de anticuerpos (si el paciente es inmunocompetente). Se estima que $\sim 30 \%$ de los chagásicos tendrían manifestaciones de la etapa crónica, lo que podría aumentar al emplear métodos diagnósticos más sensibles.

Cardiopatía chagásica crónica (CCC). Se presenta en 10 a $30 \%$ de estos pacientes, en Chile y Brasil son el $30 \%$ de los casos. Se caracteriza por su gravedad y representa la principal causa de muerte de estos enfermos. Los síntomas más frecuentes son palpitaciones y disnea de esfuerzo. La cardiopatía evoluciona a la insuficiencia cardíaca. Las arritmias son frecuentes y variadas, todos signos de mal pronóstico. El bloqueo A-V, más el bloqueo completo de rama derecha, con o sin hemi-bloqueo anterior izquierdo, son sugerentes de esta patología. Puede haber bloqueo AV completo, fibrilación auricular, bloqueo completo de rama izquierda y extrasístoles ventriculares. En corazones dilatados, se presentan fenómenos trombo-embólicos que pueden ocasionar infartos pulmonares y cerebrales. Existe fibrosis cardíaca que originan micro-aneurismas de la punta del ventrículo izquierdo.

Síntomas y signos. En un comienzo, la CCC puede ser asintomática, pero con evidentes alteraciones electrocardiografícas. Los pacientes refieren palpitaciones, disnea, lipotimia y, raramente, se encuentran soplos cardíacos.

\section{Estudio de la CCC}

- Radiografía de tórax: evidencia la presencia de cardiomegalia con o sin derrame.

- Electrocardiograma: de reposo, continuo (holter) y electrocardiograma de esfuerzo, demuestran el bloqueo completo de rama derecha, que es la alteración más frecuente de la CCC; el $40 \%$ se asocia a hemibloqueo anterior izquierdo.

- Ecocardiografía: confirma la existencia de microaneurisma de la punta, zonas de fibrosis, disminución de la contractibilidad ventricular y alteración de la fracción de eyección.

- Resonancia magnética: permite identificar daños estructurales en la pared miocárdica.

- Cintigrafía: permite observar la pared ventricular y las cavidades cardíacas.

Evolución de la CCC. La enfermedad lleva a la insuficiencia cardíaca, que junto con las arrítmias, son la causa de muerte de estos pacientes. La incidencia de la progresión de la cardiopatía chagásica es de $~ 24,8 \%$ y la de las complicaciones es de $3,5 \%$ en pacientes tratados y $16,9 \%$ en los no tratados. 
Tratamiento de la CCC. Especialmente en los estados más avanzados, la CCC es resistente a los tratamientos habituales. Los extrasístoles ventriculares pueden responder a anti-arrítmicos como la amiodarona. El trombo-embolismo requiere de tratamiento anticoagulante. En los casos de bloqueo A-V de tercer grado, es necesaria la instalación de marcapaso. En pacientes con cardiomegalia importante e insuficiencia cardíaca congestiva refractaria a las terapias habituales, se ha indicado, como una solución, el trasplante cardíaco. Ayuda en esta condición el tratamiento etiológico (ver Parte VI: Tratamiento de la enfermedad de Chagas).

\section{Megasíndromes}

Esofagopatía chagásica. Conocida como megaesófago, aperistalsis o acalasia del esófago. Se diagnostica, frecuentemente, antes de los 40 años de edad. El esófago se presenta dilatado en diferentes grados y más tardíamente elongado (dolicomegaesófago). Tiende a la hipertrofia de las capas musculares y en la mucosa se producen paraqueratosis. Microscópicamente existe destrucción de las neuronas parasimpáticas con áreas de inflamación crónica, lo que ocasiona la pérdida progresiva de la coordinación motora y de la capacidad contráctil en la manometría esofágica. Luego aparece disfagia, dolor y regurgitación. La disfagia es el síntoma principal, que puede ser alternada entre lógica e ilógica. El paciente presenta odinofagia, regurgitación, eructos y pirosis. Es posible que se asocie con megacolon o cardiopatía.

Diagnóstico. Los antecedentes epidemiológicos son importantes. Las manifestaciones clínicas, radiología y manometría esofágica permiten efectuar el diagnóstico. Es conveniente realizar endoscopia digestiva alta para descartar la presencia de cáncer.

Tratamiento. Medidas para facilitar el vaciamiento del esófago: dilataciones neumáticas, toxina botulínica, cardiomiotomía clásica o por laparoscopia.

Para tratamiento específico, ver Parte VI: Tratamiento de la enfermedad de Chagas.

Colopatía chagásica. La colopatía se presenta entre los 40 y 50 años de edad. Se produce una disfunción motora de los segmentos del colon, por denervación parasimpática intramural. La alteración toma, preferentemente, el sigmoides y el recto, originando una dilatación de esa zona. La expresión clínica básica del megacolon es la constipación progresiva con dificultad para la evacuación. El enfermo suele utilizar laxantes, y enemas y consultar tardíamente. En estos cuadros clínicos se producen períodos de distensión abdominal y, secundariamente, abdomen prominente. Se puede palpar un fecaloma en la fosa iliaca izquierda o directamente al realizar un tacto rectal. Las complicaciones del megacolon chagásico son el vólvulo y la obstrucción intestinal por fecaloma.

Diagnóstico. Son importantes los antecedentes epidemiológicos, la sintomatología, el estudio con una radiografía de abdomen simple, enema baritada y colonoscopia. Para el diagnóstico etiológico, los exámenes parasitológicos directos e indirectos son fundamentales (ver Parte V: Diagnóstico de la Enfermedad de Chagas).

Tratamiento del megacolon

- Quirúrgico: recto y sigmoidectomía.

- Tratamiento etiológico (ver Parte VI: Tratamiento de la enfermedad de Chagas).

\section{Enfermedad de Chagas en la infancia y adolescencia}

\section{Enfermedad de Chagas congénita}

Esta presentación es consecuencia de una transmisión del parásito durante el embarazo, ya sea en etapa aguda o crónica de la enfermedad. Constituye el 10\% de los casos seropositivos en la infancia y adolescencia. Sesenta por ciento de los recién nacidos hijos de madres chagásicas son seropositivos, debido al traspaso de anticuerpos pasivos maternos y se negativizarían antes del año de edad. Se desconoce el número de niños que adquieren la infección por vía parenteral (drogadicción parenteral, trasplante de órganos o transfusiones) y vectorial.

La prevalencia de la infección chagásica en mujeres embarazadas en Sudamérica, varía según las zonas estudiadas de 2 a $51 \%$ en zonas urbanas y de 23 a $81 \%$ en ambientes rurales, dependiendo del linaje de la cepa de $T$. cruzi. La madre se puede encontrar en cualquier etapa de la infección al momento de embarazarse. En etapa aguda de la infección existe una intensa parasitemia y, por lo tanto, mayor riesgo de transmisión. Estas gestaciones pueden terminar en aborto, mortinato, prematuro o un recién nacido enfermo. En ocasiones, el RN es asintomático. En las etapa crónica indeterminada y determinada hay menor carga de parasitemia, existiendo, por lo tanto, riesgo de transmisión, con iguales consecuencias que las ya descritas.

En Chile, la frecuencia de transmisión del $T$.cruzi de madre a hijo se estima que es inferior al $10 \%$, pero aún faltan estudios para corroborar estas cifras. Estudios realizados en otros países de Latino América dan cuenta de cifras que varían entre 0,5 y $10,4 \%$. 




Tripanosoma cruzi alcanza la circulación fetal por vía hematógena, como resultado de una placentitis, donde se encuentran focos inflamatorios agudos y/o crónicos, áreas de necrosis, presencia de células gigantes y parasitismo de las células trofoblásticas y de los macrófagos, constituyendo cuadros de vellositis e intervellositis de intensidad variable; también el parásito puede penetrar en forma activa hacia la circulación fetal. No existe una correlación directa entre el grado de parasitismo placentario e infección fetal.

Puede existir infección congénita en embarazos sucesivos, como así también en gemelos, incluso se ha descrito infección congénita de segunda generación. En general, la mayoría de los $\mathrm{RN}$ infectados nacen asintomáticos (70 a 80\%). El RN sintomático presenta manifestaciones clínicas similares a otras etiologías del síndrome de TORCH y debe considerarse esta infección dentro del diagnóstico diferencial de este síndrome.

El RN puede ser prematuro o de término, pequeño para la edad gestacional, destacando en la signología: hepato-esplenomegalia, ictericia, anemia, neumonía intersticial, compromiso variable del SNC (que puede manifestarse incluso sólo por alteraciones citoquímicas en el LCR), miocarditis, compromiso del fondo de ojo y de la piel. La ausencia de síntomas al nacer no implica ausencia de infección y de enfermedad a futuro; por el contrario, ese niño puede presentar, al igual que en la
Figura 1. Diagnóstico de la infección transplacentaria por Trypanosoma cruzi. 
forma adquirida vectorialmente, meses o años después, manifestaciones de la etapa crónica de la enfermedad.

La posibilidad de una infección concomitante por $T$. cruzi y VIH en RN hijos de madres portadoras de ambas infecciones, agrava la evolución de estos pacientes, como ya se ha descrito en Argentina y otros países.

El diagnóstico de la infección se realiza a través de la detección de anticuerpos específicos en la mujer embarazada y por métodos directos en RN hijos de madres chagásicas (Figura 1).

\section{Formas de transmisión no vertical}

Las otras formas de presentación agudas de transmisión no vertical no difieren de lo descrito en enfermedad de Chagas en pacientes adultos (ver párrafos correspondientes).

\section{Diagnóstico de laboratorio}

Detección de anticuerpos específicos. IFI para detección de IgG e IgM, ELISA IgG e IgM utilizados para el diagnóstico de rutina.

Detección del parásito. Métodos directos:

- Frotis sanguíneo.

- Examen de sangre entre lámina y laminilla.

- Gota gruesa.

- Método de Strout (microhematocrito).

- RPC, técnica de biología molecular que tiene gran sensibilidad y ha reemplazado, en la mayoría de los casos, al xeno-diagnóstico. Idealmente, esta técnica debería realizarse a tiempo real, lo que permite una cuantificación de la parasitemia.

- En esta presentación el xeno-diagnóstico tiene poca utilidad, el que dependerá de la gravedad del niño, ya que el resultado tiene una demora de entre $30 \mathrm{y}$ 90 días.

\section{Tratamiento}

Ver Parte VI: Tratamiento de la enfermedad de Chagas.

\section{Resumen}

Se describen las distintas etapas de la enfermedad en el adulto: aguda, indeterminada o latente y crónica. Se menciona cómo se realiza el diagnóstico etiológico y la indicación de tratamiento. En la etapa crónica, cuando existe compromiso cardíaco así como también digestivo (esófago o colon), se mencionan los síntomas y signos, la evolución, los exámenes de apoyo diagnóstico y el tratamiento. Se analiza la enfermedad de Chagas congénita desde su prevalencia en la embarazada, la etapa de la enfermedad en la madre, las repercusiones de la transmisión del parásito sobre el producto de la concepción, la frecuencia de la transmisión, cómo se produce la infección del feto, la situación en embarazos sucesivos, hasta las consecuencias sobre el recién nacido, incluyendo la sintomatología cuando éste nace enfermo. Se comenta la concomitancia con la infección por virus de inmunodeficiencia humana. Las formas de presentación no vertical en la infancia y adolescencia no difieren de la enfermedad de Chagas en el adulto. Se menciona el diagnóstico directo e indirecto de la infección y se presenta un algoritmo del diagnóstico y seguimiento de la infección vertical por Trypanosoma cruzi.

\section{Referencias}

1.- Arribada A, Apt W, Aguilera X, Solari A, Arribada A, Sandoval J. Chagas cardiopathy in the first region of Chile. Clinical epidemiological and parasitologic study. Rev Méd Chile 1990; 118: 846-54

2.- Auger S R, Storino R, De la Rosa M, Caravello A, González M I. Chagas y SIDA. Rev Arg Cardiol 2005; 73: 439-45.

3.- Bonamatti A, Castelo F, Ramos L. Infeccao por $T$ cruzi em candidatos a doador de sangue. Rev Saúde Pública 1998; 32: 566-71.

4.- Chagas C. Una nueva entidad mórbida: informe de estudios etiológicos y clínicos en El desafío de la Epidemiología problemas y lecturas seleccionadas. Organización Panamericana de la Salud Publicación Científica 505, 1991; 83-4.
5.- Contreras M, Rivera J, Schenone H, et al. Enfermedad de Chagas en Chile. Sectores urbanos X Frecuencias de infección chagásica en donantes de sangre y en madres y recién nacidos de las ciudades de La Serena, Ovalle e Illapel IV Región, 1983-1985. Bol Chil Parasitol 1985; 40: 72-6.

6.- Corti M. AIDS and Chagas disease. AIDS Patient Care. 2000; 14: 581-7.

7.- Corti M, Yampolsky C. Prolonged survival and immune reconstitution after chagasic meningo-encephalitis in a patient with acquired immunodeficiency syndrome. Rev Soc Bras Med Trop 2006; 39: 85-8.

8.- Da-Cruz A M, Igreja R P, Dantas W, Junqueira A C, Pacheco R S, Silva Goncalves A J, et al. Long term follow-up of co-infected HIV and Trypanosoma cruzi Brazilian patients. Trans R Soc Trop Med
Hyg 2004: 98: 728-33.

9.- Del Castillo M, Mendoza G, Oviedo J, Pérez Blanco R P, Anselmo A E, Silva A M. AIDS and Chagas disease with central nervous system tumor-like lesion. Am J Med 1990; 88: 693-4.

10.- Fasano M B. Inmunodeficiencias primarias. Consideraciones clínicas sobre las infecciones en pacientes inmunodeprimidos. Secretos de la Infectología. Mc Grill Interamericana 1998.

11.- Ferreira M S, Nishioka S, Rocha A, Silva A M, Ferreira R G, Olivier W, et al. Acute fatal Trypanosoma cruzi meningoencephalitis in a human immunodeficiency vírus-positive hemophiliac patient. Am J Trop Med Hyg 1991; 45: 723-7.

12.- Ferreira M S, Nishioska S, Silvestre M T, Vorges A S, Nunes Araujo F R, Rocha A. 
Reactivation of Chagas disease in patients with AIDS: report of three new cases and review of the literature. Clin Infect Dis 1997; 25: 1397-400.

13.- Freilij H, Altchen J. Congenital Chagas disease diagnostic and clinics aspects. Clin Infect Dis 1995; 21: 551-5.

14.- García A, Bahamonde M, Verdugo S, Correa J, Pastene C, Solari A, et al. Infección transplacentaria por Trypanosoma cruzi. Rev Méd Chile 2001; 129: 330-2.

15.- Harms G, Feldmeier H. Review: HIV infection and tropical parasitic diseasesdeleterious interactions in both directions? Trop Med Intern Health 2002, 7: 479-88.

16. - Ferreira M S, Nishioka S de A, Silvestre M T, Borges A S, Nunes-Araujo F R, Rocha A. Reactivation of Chagas' disease in patients with AIDS: report of three new cases and review of the literature. Clin Infect Dis 1997; 25: 1397-400.

17.- Lages-Silva E, Ramírez L E, Silva-Vergara M L, Chiari E. Chagasic meningoencephalitis in a patient with acquired immunodeficiency syndrome: diagnosis, follow-up, and genetic characterization of Trypanosoma cruzi.
Clin Infect Dis 2002; 34: 118-23.

18.- WHO 2002. Control of Chagas disease. WHO Technical Report Series N-905 Geneva.

19. - Lorca M, Bahamonde M I, García A, Tassara R, Urarte E, Contreras M del C, et al. Trypanosome cruzi transplacental infection in Chile: diagnosis, treatment and control. Rev Soc Bras Med Trop 2005; 3852: 46-8.

20.- Oddo D, Casanova M, Acuña G, Ballesteros J, Morales B. Acute Chagas disease (Trypanosomiasis Americana) in acquired immunodeficiency syndrome: report two cases. Hum Pathol 1992; 23: 41-4.

21.- Pérez Bianco R, Anselmo A, Soria A, Ruibal Ares B, Bouza MB, Muchinik G, et al. HIV-1 infection in patients with hemophilia. The Argentinian experience from 1983 to 1990. Medicina B Aires 1992; 52: 3-9.

22.- Rivera J, David L, Levine B. Reactivation of cardiac Chagas disease in acquired immune deficiency syndrome. Am J Card 2004; 94: 1102-3.

23.- Schijman A G, Vigliano C, Burgos J, Favoloro R, Perrone S, Laguens R, et al. Early diagnosis of recurrence of $T$. cruzi infection by polymerase chain reaction after heart transplantation of chronic Chagas heart disease patient. J Heart Lung Transplant 2000; 19: 1114-7.

24.- Schmunis G. La tripanosomiasis americana como problema de salud pública, en la Enfermedad de Chagas y el Sistema Nervioso OPS, Publicación Científica 547, 1994.

25.- Schwint O, Turconi, P, Cazzulo A, Magagnin S, Grana D, Seminovich M, et al. Compromiso cardíaco en el SIDA pediátrico. Rev Arg 2002; 70: 101-7.

26.- Shearer W, Rosenblatt H, Gelman R, Oyomopito R, Pleager S, Stiehm R, et al. Lymphocyte subsets in healthy children from birth through 18 years of age: The Pediatric AIDS Clinical Trials Group P1009 study. J Allergy Clin Immunol 2003; 112: 973-80.

27.- Stiehm E R. Immunologic Disorders in Infant and Children, 4Th ed. Philadelfia WB Saunders, 1996.

28. - Umezawa E, Simonsen A N, Corbett C. Chagas disease. Lancet 2001; 357: 797-9

29.- Vaidian A K, Weiss L M, Tanowitz H B. Chagas' disease and AIDS. Kinetoplastid Biol Dis 2004; 3: 2. 